\title{
Upregulation of human PINK1 gene expression by NFKB signalling
}

\author{
Xiaoling Duan ${ }^{1,2}$, Jade Tong ${ }^{2}$, Qin Xu ${ }^{2}$, Yili Wu ${ }^{1,2}$, Fang Cai ${ }^{2}$, Tingyu $\mathrm{Li}^{1}$ and Weihong Song ${ }^{1,2^{*}}$
}

\begin{abstract}
Parkinson's disease (PD) is one of the major neurodegenerative disorders. Mitochondrial malfunction is implicated in PD pathogenesis. Phosphatase and tensin homolog deleted on chromosome 10 (PTEN)-induced putative kinase 1 (PINK1), a serine/threonine kinase, plays an important role in the quality control of mitochondria and more than 70 PINK1 mutations have been identified to cause early-onset PD. However, the regulation of PINK1 gene expression remains elusive. In the present study, we identified the transcription start site (TSS) of the human PINK1 gene using switching mechanism at 5'end of RNA transcription (SMART RACE) assay. The TSS is located at 91 bp upstream of the translation start site ATG. The region with $104 \mathrm{bp}$ was identified as the minimal promoter region by deletion analysis followed by dual luciferase assay. Four functional cis-acting nuclear factor kappa-light-chain-enhancer of activated B cells (NFKB)-binding sites within the PINK1 promoter were identified. NFKB overexpression led to the up-regulation of PINK1 expression in both HEK293 cells and SH-SY5Y cells. Consistently, lipopolysaccharide (LPS), a strong activator of NFKB, significantly increased PINK1 expression in SH-SY5Y cells. Taken together, our results clearly suggested that PINK1 expression is tightly regulated at its transcription level and NFKB is a positive regulator for PINK1 expression.
\end{abstract}

Keywords: PINK1, Mitochondrial function, NFkB, Transcription

\section{Background}

Parkinson's disease (PD) is the second most common neurodegenerative disease [1]. PD patients commonly suffer from muscular dysfunction which resulted in body rigidity, tremors, bradykinesia, posture instability, and Parkinsonian gait. Secondary symptoms such as anxiety, depression, memory loss, and dementia may appear over the course of disease progression [2,3]. The pathological hallmarks of PD include neurodegeneration of dopaminergic neurons specifically in the substantia nigra [3], and intracellular aggregation of misfolded proteins forming lewy bodies [4]. Although clinical and experimental studies suggest the involvement of protein misfolding, oxidative stress and mitochondrial dysfunction, the fundamental cause of the disease, its underlying mechanism remains elusive.

\footnotetext{
* Correspondence: weihong@mail.ubc.ca

${ }^{1}$ Chongqing City Key Lab of Translational Medical Research in Cognitive Development and Learning and Memory Disorders, and Ministry of Education Key Lab of Child Development and Disorders, Children's Hospital of Chongqing Medical University, Chongqing 400014, China

${ }^{2}$ Townsend Family Laboratories, Department of Psychiatry, Brain Research Center, The University of British Columbia, 2255 Wesbrook Mall, Vancouver, BC V6T 1Z3, Canada
}

\section{() Biomed Central}

Phosphatase and tensin homolog deleted on chromosome 10 (PTEN)-induced putative kinase 1 (PINK1), initially was identified as a downstream molecule of PTEN in cancer cells [5]. PINK1 is a type I transmembrane protein of 581 amino acids with a putative serine/threonine kinase domain and an $\mathrm{N}$-terminal mitochondrial targeting signal. Upon synthesis, the full-length PINK1 (FL-PINK1) is imported into mitochondria with the Cterminus facing the cytosol and undergoes proteolysis to produce a predominant product of $\sim 55 \mathrm{kDa}(\Delta 1$-PINK1) and a minor one of $\sim 45 \mathrm{kDa}(\Delta 2$-PINK1) [6,7]. The proteolytic process is mediated by matrix processing peptidase and presenilin-associated rhomboid-like protein (PARL) [8]. $\triangle 1$-PINK1 was reported to be released to the cytosol and interacts with Parkin, a PD-associated E3 ubiquitin ligase [9]. The binding of $\triangle 1$-PINK1 with Parkin impairs the recruitment of Parkin to mitochondria and leads to the degradation of Parkin and PINK1 by proteasome pathway in healthy mitochondria [9]. Parkin, PINK1 and DJ-1 formed a complex to promote ubiquitination and degradation of Parkin substrates, including Parkin itself and Synphilin-1 [10]. However, in 
dysfunctional mitochondria with reduced mitochondrial membrane potential and mitochondrial oxidative stress, full-length PINK1 is aggregated on the mitochondrial membrane and recruits Parkin to mitochondria through the phosphorylation of Parkin, resulting in mitochondrial autophagy. PINK1 deficiency reduces mitochondrial membrane potential and compromises complex I activity of the mitochondria respiratory chain [11]. Studies suggest that PINK1 together with Parkin is critical for the quality control of mitochondria, including mitochondrial integrity, turnover, and functions. More than 70 mutations in the PINK1 gene were identified in familial PD in an autosomal recessive manner [12,13]. Most of these mutations fail to phosphorylate and recruit Parkin to mitochondria, leading to the accumulation of dysfunctional mitochondria and eventually neuronal death. This strongly suggests that PINK1 plays a critical role in PD pathogenesis and dysregulation of PINK1 may contribute to the development of PD.

Nuclear factor kappa-light-chain-enhancer of activated B cells $(\mathrm{NFKB})$ is a family of diametric transcription factors, regulating numerous genes involving in cell survival, inflammation and immunity. The NFkB family consists of five members, p50, p52, p65 (RelA), RelB and C-Rel. All of them share an N-terminal Rel homology domain (RHD) responsible for DNA binding and homoor hetero-dimerization. Only three members including p65, RelB and C-Rel contain transcription activation domain (TAD) necessary for the activation of target genes. $N F K B$ exists in forms of homo- or hetero-dimer and the most abundant form is the p65/p50 heterodimer. NFkB dimers bind with inhibitor of $\kappa B(\mathrm{I} \kappa \mathrm{B})$ proteins in cytoplasm, preventing its nuclear translocation and DNA binding. Once IKB protein degradation is induced, $\mathrm{NF}_{\kappa} \mathrm{B}$ dimers are released from IкB-NFkB complex and subsequently translocated from cytoplasm to nucleus [14], thus transcription regulation of its target genes is initialized [15]. NFKB can be activated by stimuli such as oxidative stress, cytokines, free radicals and biological antigens. Oxidative stress or free radicals has been shown to be involved in PD. An increase of NFKB translocation to the nucleus and activation have been observed in dopaminergic neurons from both PD animal models and patient samples as well as other neurodegenerative disorders [16-21]. It has been shown that NFKB plays an important role in regulating PD related genes, such as UCHL1 [22], USP24 [23] and RNF11 [24].

Although the function of PINK1 is well studied in terms of mitochondrial quality control, the regulation of PINK1 gene expression is barely explored. In the present study, we aimed to understand the transcriptional regulation of the PINK1 gene. For the first time, we identified that the transcription start site (TSS) of PINK1 is located $91 \mathrm{bp}$ upstream of the translation start site
(ATG). The region of 104 bp $(-78$ to +26$)$ contains the minimal promoter with functional transcriptional activity for the PINK1 gene. Furthermore, we showed that the PINK1 gene promoter contains 4 functional cis-acting NFkB-binding sites, which promote PINK1 expression. Taken together, our results demonstrated that PINK1 expression is tightly regulated at its transcriptional level and that $\mathrm{NFKB}$ is a positive regulator for PINK1 expression.

\section{Results and discussion}

\section{Cloning the human PINK1 gene promoter and mapping} its transcription initiation site

The human PINK1 gene spans a region of 18,056 bp on chromosome 1p36. The human PINK1 gene transcript (NCBI Reference Sequence: NM_032409) is 2680-bp long and composed of 8 exons (Figure 1A). Human genomic DNA samples were extracted from SH-SY5Y cells and an 1825 bp 5' flanking region of the PINK1 gene was amplified and cloned. The DNA fragment was sequenced (Figure 1B). To identify the transcription start site of human PINK1 gene, SMART-RACE was performed. The 5'ends of PINK1 cDNA with approximate $650 \mathrm{bp}$ in length were amplified (Figure 1C). The sequencing results indicated that the transcription start site (TSS), an adenine labeled as +1 , is located 91 bp upstream of the translation start site (Figure 1D). Further sequence analysis and a computer-based transcription factor binding site search using Genomatix and TFSearch revealed that the human PINK1 gene has a complex transcriptional unit. The human PINK1 gene promoter lacks typical TATA and CAAT boxes, but contains several putative regulatory elements, such as AP1, MEF, and cAMP-responsive elements (Figure 1B).

\section{Functional characterization of the PINK1 promoter}

To determine the functional promoter region of PINK1 gene, we sub-cloned the 1,825 bp of 5 ' flanking region of the PINK1 gene into the promoterless plasmid vector pGL3-basic. The pGL3-basic vector lacks eukaryotic promoter and enhancer sequences upstream of a reporter luciferase gene. The luciferase activity in cells transfected with this plasmid depends on the presence and proper orientation of a functional promoter upstream of the luciferase gene. The pPINK1-A plasmid was constructed to contain the 1,825 bp fragment from -1799 to +26 bp of PINK1 gene upstream of the luciferase reporter gene. Plasmid DNA was transfected into HEK293 cells, and luciferase activity was measured by a luminometer to reflect promoter activity. Compared with cells transfected with an empty vector pGL3-basic, pPINK1-A-transfected cells showed a robust luciferase activity $(113.47 \pm 10.63 \mathrm{RLU})$ (Figure 2B). This result indicated that the $1,825 \mathrm{bp}$ fragment contains the functional promoter region of the human PINK1 gene. To further identify the minimal 


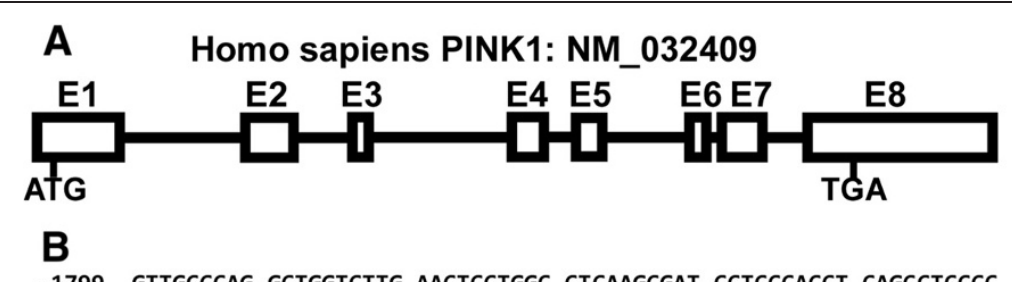

-1799 GTTGCCCAG GCTGGTCTTG AACTCCTGGC CTCAAGCGAT CCTCCCACCT CAGCCTCCCC

-1740 ACGTGCTGGA GTGAACCACT GCAAACCTGG CTTCAGACGA GTGGATTTAA ACTTTAAATC

-1680 CACTCATCTG TGCCCCAACT AAGTTTCCAT GAGTGAGCCT GTGGTTACTG GAATGTGAGA AP1F

-1620 TTTCTCCAAC TTTCTTTCCC TTATCATtCT TCAAATtACA ACtTCAGTGG AGCATtTAAA

-1560 ATTATAATTA AAAATTCCCT GAGGTTGATC CAAAAAATTA AATATCTGAT ATTCATGTGC

-1500 CTCTATACAA ATGGGAAATT CATCTATGCC TCTCCCAAAT AAATATGATT GGATATCACA NFKB

-1440 AGAACCATAA AATTCCTCCC TTAAGgAaga TTATGAGAGA GACCAGCACA CACACACACA -1380 GTGCAAGCTA AAGCAACCCC GTCTTGGATG CTAATCCACC AAGTTGATGT GTTTTGTTGG

-1320 TTTTTTGGTT TTGTTTTGAG ACAGAGTCTC ACTCTGTCGC CCAGGATAGA GTGCAGTGGT

-1260 GTGATCTTTG CTCACTACAA CTTCCACCTC CTGGGTTCAA GCGATTCTCC TGCCTTAGCC

-1200 ACCTGAGTAG CTGGGATTAC AGGCGTGTGC CACCACGCCC AGCTAGTTTT TGTATTTTTA MEF3

-1140 GTAGAGATGG TGTTTTGTTG GCCAGGCTGG TCATGAACTT CCGGACTCAA GTGATCCACC

-1080 TGCCTCAGTC TCCCAAAATG CTGGGATTAC AGGCTGTGAG CTACCACACC CGGCCAACCA

-1020 AGTTGACTTC TGATTAACCA GCTTTTTAGGG AAGGCCTCTA AGATTTCCAG TTTATCTATI

-960 GITCCTTGTG TAAAAGTATG TACTTACCAT AAATCCTGCC CTTAGGCAGA TTCACACAGC CREB

-900 ATTCTTGCCT TTCCCTGGGG GACTGACTTC AAATGTCCGT CACATTCCTT TCCTATAGCA CREB

-840 TATAGGCCCT GGGTCTTGGG GGTAATGGCA TGGGGATCCA CCATCTTGTC TCCCTGCCGC NFKB

-780 TGAAGCCAGA GACTATGGCT TCTGTTCATA AATCCCTTTC TCCITAAATA TGAAGTCAAA CREB

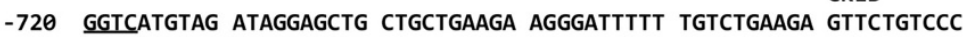

-660 CTGGGCTTAC TTGGCTATGG GGTGGACCCC TGGCCAGGAG ACAGCAAACT GTTTCAGTAA

-600 TGTGCGTGTC GTGCGTGTGI GTGTTCTGTG GTGAGCAGGC CTATGCCATT AAACAAACGG CREB

-540 TGTGGCTTTG GGCAAGCTGC TATCTTGGGA CCTCAGCACA CTCATTCATA AATTGTGTCT

-480 TTTGGGTGAG ATTTGTCTTG GGGTCTTCAA AACCCCTGAG ACTGTGGACA CATACAGCAG

-420 CCTAACGGCA TTGAGGAGGG CAAGCATTCA ACAGTTAGGA CAATGTGAAC TGTAGCTCAG

-360 CTCTGCTAGG TACCTTCACA AGACCTCGAA TGCTGCCCCT TACTATGCCT CGGTTTTCTT

-300 ATCTATAAAA ACGGCATTTT TATCTTGTTG GTGGAGCTGT TAAATAAATT AAAAGACGTA CREB

-240 AAGGGTCTGG CACCATGGTT GGCAAAAAAT ATCAGTTTCC CTTCTCGACT TCTCGATTTT

-180 GCCCAGGACC AGTGATGTTC ACATTCAGGA CCTGCCTGAA CCGGCAAGCC CTCCACGTGG

-120 GTCCAAAGTG CAAAGGGAAA GICACTGCIA GAGGCGCCAG TACCAGCATA GCGCCCCCAC NFKB

-60 GCGCCGAGTC GGGGAACTGC CGCGGGGGCC GGCCCCGCCC ACCAGCGCCT GCGCCTGCGC NFKB

+1 AGAGGCACCG CCCCAAGTTT GTTGTGACCG GCGGGGGACG CCGGTGGTGG CGGCAGCGGC

+61 GGCTGCGGGG GCACCGGGCC GCGGCGCCAC CATGGCG

C



Figure 1 Sequence features of the human PINK1 gene promoter. (A) The genomic organization of human PINK1 gene on chromosome 1. E represents exon. ATG is the translation start codon and TGA is the stop codon. (B) The nucleotide sequence of the human PINK1 gene from -1799 to $+97 \mathrm{bp}$. The adenine +1 represents the transcription start site. The putative transcription factor binding sites are underlined in bold face. (C) SMARTer-RACE was performed to map the PINK1 transcription start site. The PCR product was run on a 1.5\% agarose gel. (D) The PCR product was cloned into PCDNA4 vector and sequenced to identify the transcription start site. The first base after the adapter is the TSS from which is underlined.

promoter region required for PINK1 gene expression, a series of deletion mutants within the 1,825 bp fragment of pPINK1-A were generated as indicated in Figure 2A. The luciferase activity assays of these deletion mutants were performed. The results indicated that placing the $1,825 \mathrm{bp}$ fragment in a reverse orientation (pPINK1-B) completely abolished luciferase activities observed in pPINK1A transfected cells (Figure 2B). Deletion of -1799 to $103 \mathrm{bp}$ in pPINK1-F reduced the luciferase activities of pPINK1-A moderately (71.16 $\pm 3.02 \mathrm{RLU})$. Deletion of the 


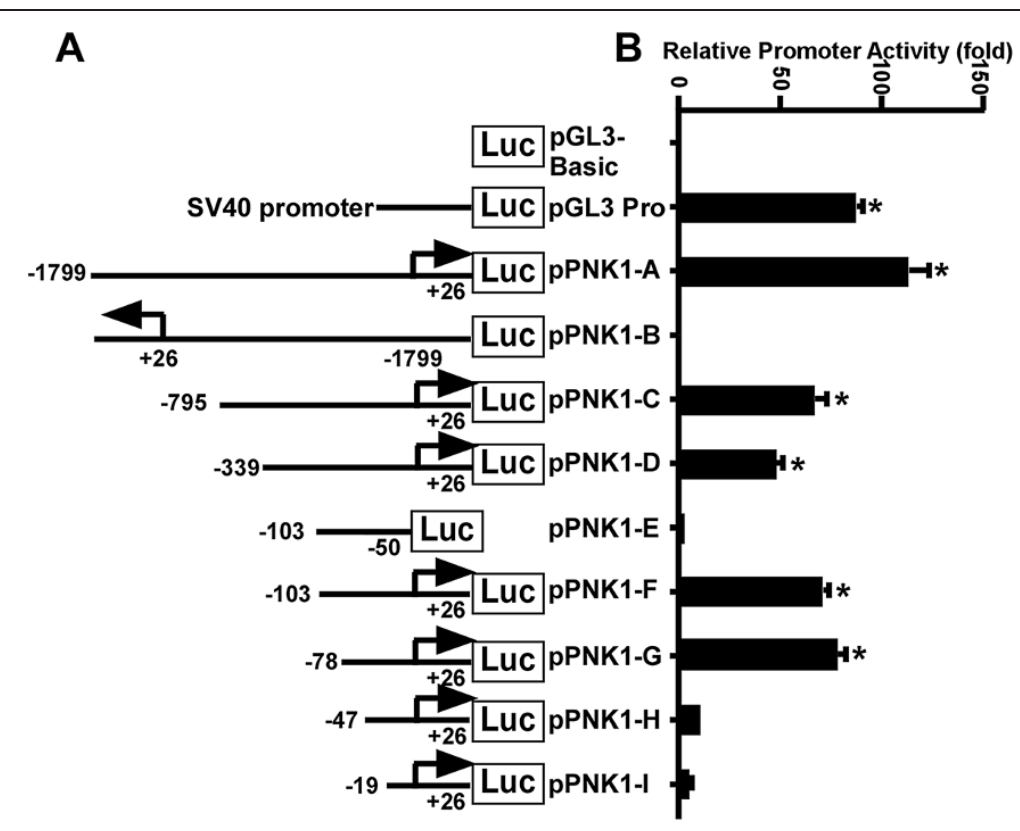

Figure 2 Deletion analysis of the human PINK1 gene promoter. (A) Schematic diagram of the PINK1 promoter constructs consisting of the $5^{\prime}$ flanking region with serial deletions cloned into the pGL3-basic vector. Arrow shows the direction of transcription. The numbers represents the end points of each construct. (B) The deletion plasmids were cotransfected with pCMV-Luc into HEK293 cells. $24 \mathrm{~h}$ after the transfection, the luciferase activity was measured and expressed in relative luciferase units (RLU). The pCMV-Luc was used to normalize for transfection efficiency. The values represent means \pm SEM. $n=3,{ }^{*} p<0.0001$, by one-way ANOVA followed by post hoc Tukey's multiple comparisons test.

identified transcription initiation site (pPINK1-E) from pPINK1-F occluded the remaining luciferase activities (Figure 2B). Further deletion of the promoter region to -78 to +26 bp maintained similar luciferase activity in pPINK1-G (78.44 $\pm 4.74 \mathrm{RLU})$ comparing to pPINK1-F $(p>0.05)$. However, additional deletion of $31 \mathrm{bp}$ from pPINK1-G to pPINK1-H $(-47$ to +26$)$ significantly reduced the luciferase activity to $11.46 \pm 2.50 \mathrm{RLU}$ $(p<0.05)$. These data suggest that the region from -78 to +26 , containing the transcription start site, possesses the minimal promoter activity.

\section{The human PINK1 gene promoter contains NFKB binding sites}

Computer-based transcription factor binding site analysis revealed four putative NFKB elements in the $1.8 \mathrm{~kb}$ promoter region of the human PINK1 gene (Figure 1B). To determine whether NFKB signaling regulates PINK1 gene transcription by interacting with these putative $\mathrm{NFK}_{\mathrm{K}} \mathrm{B}$ cis-acting elements, the effect of $\mathrm{NFK}_{\mathrm{K}} \mathrm{B}$ overexpression on the promoter activity of the $1.8 \mathrm{~kb}$ region were examined. Plasmid pPINK1-A, containing 4 NFKB binding sites, was transfected into HEK293 cells, SHSY5Y cells or N2A cells with either the NFKB expression plasmid or the empty vector pMTF. The results showed that $\mathrm{NF}_{\mathrm{K} B} \mathrm{~B}$ overexpression significantly increased PINK1 promoter activity to $3.43 \pm 0.55$ folds (Figure $3 \mathrm{~A}$ ), $2.11 \pm$ 0.10 folds (Figure 3B) and $1.63 \pm 0.01$ folds (Figure 3C) in HEK293, SH-SY5Y cells and N2A cells, respectively $(p<0.01)$. These results suggest that NFKB activates PINK1 gene transcription and the PINK1 promoter fragment contains functional NFKB sites.

The four putative NFkB elements in the 1.8-kb PINK1 promoter region are located at -1493 to $-1474 \mathrm{bp},-814$ to $-794 \mathrm{bp},-111$ to $-92 \mathrm{bp}$, and -54 to $-35 \mathrm{bp}$, respectively. The sequences of these regions are highly homologous to the NFKB consensus sequence 5'-GGGRNNYYCC in which $\mathrm{R}$ stands for purine, $\mathrm{Y}$ stands for pyrimidine, and $\mathrm{N}$ stands for deoxynucleotides. To determine which putative binding site actually binds with NFkB, EMSA was performed using four pairs of synthesized oligonucleotides containing the binding sequence. The sequences of these four oligonucleotides are: PINK1-NFkB-1, 5'- caaatgggaaattcatctat, PINK1-NFKB-2, 5'- ggcatggggatccaccatctt, PINK1-NFKB-3, 5'- gcaaagggaaagtcactgct, and PINK1NFKB-4, 5'- agtcggggaactgccgcggg, respectively. A NFкB binding probe (NFKB-Wt-oligo) served as a positive binding probe and a mutant $\mathrm{NFKB}$ binding probe (NFKB-Mu-oligo) which loses the NFKB binding ability served as a negative binding probe [25]. NFkB-Wt-oligo was labelled with IRDye-700 and the labelled probe was named NFkBwt-IR700. After 30-min incubation with NFKB enriched nuclear extracts of HEK293 cells, the NFKBwt-IR700 bound to NFkB protein and formed a shifted DNA-protein complex band (Figure 3D, lane2). Adding 10-fold of the unlabelled NFKB-Wt-oligo sharply 


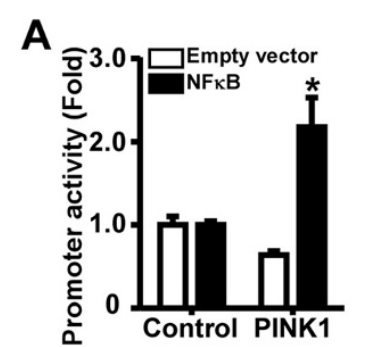

\section{B}

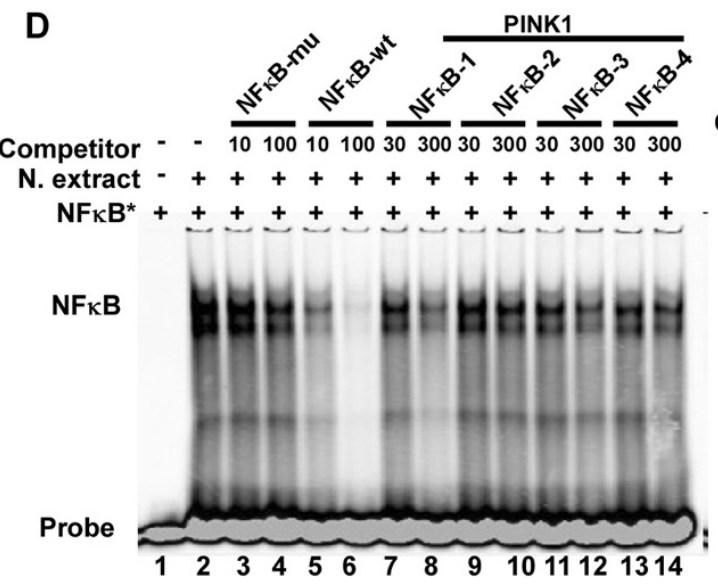

C
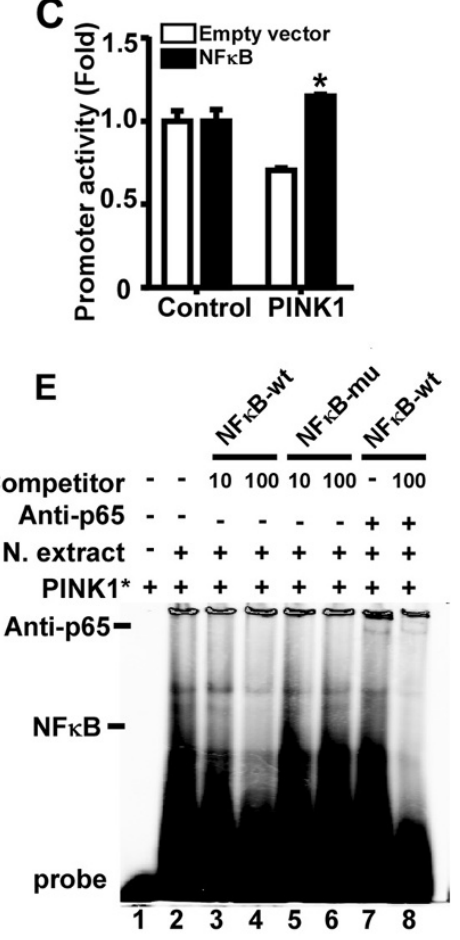

Figure 3 NFKB binds to human PINK1 gene promoter. The effects of NFKB on the human PINK1 promoter in HEK293 cells (A), SH-SY5Y cells (B) and N2A cells (C) were analyzed by luciferase reporter assays. The PINK1 promoter reporter plasmid (pPINK1-A) or pGL3-promoter was co-transfected into cells with NFKB expression plasmid or the empty vector pMTF. Values indicate means \pm SEM. $n=3,{ }^{*} p<0.01$ by Student's $t$-test.(D) EMSA with NFKB p65 consensus probe. Lane 1 is labeled probe alone without protein extract. Incubation of the probe with NFkB p65 enriched nuclear extracts forms a shifted DNA-protein complex band (lane 2). Competition assays were performed by further adding different competition oligonucleotides including NFkB-mu, NFkB-wt, and PINK1-NFkB. (E) EMSA with PINK1 NFkB-1 probe. Lane1 is labelled IR700-PINK1NFkB-1 probe only. Addition of NFKB p65 enriched nuclear extracts forms a shifted DNA-protein complex band (lane 2). Lane3 to 6, the competitor NFKB-wt and NFKB-mu were added at 10- or 100-fold. Lane 7 and 8, addition of anti-p65 antibody forms a super shifted DNA-proteinantibody band (lane7) and NFkB-wt was added for competition (lane 8).

reduced the intensity of the shifted band, indicating that the unlabelled NFKB-Wt-oligo competed with and reduced the binding of NFkB-wt-IR700 to NFkB protein (Figure 3D, lane 5). Increasing the amount of unlabelled NFKB-Wt-oligo to 100-fold almost completely abolished the shifted band (Figure 3D, lane 6). Addition of the NFkB-Mu-oligo, which fails to bind with NFkB, did not affect the interaction between NFKBwt-IR700 and protein (Figure 3D, lane 3-4). The PINK-NFkB-1 to -4 oligos were tested for their ability to compete against NFKBwtIR700 binding with NFKB protein. The results indicated that addition of 30-fold excess of the four PINK1-NFKB oligos individually barely reduced the intensity of the shifted band (Figure 3D, lane 7, 9, 11, 13). However, increasing the amount to 300 -fold significantly reduced the intensity of the shifted band for PINK1-NFKB-1 and PINK1-NFkB-3, with PINK1-NFkB-1 being a stronger competitor than PINK1-NFKB-3 in binding with $\mathrm{NFKB}$ protein (Figure 3D, lane 8 and 12). PINK1-NFKB-2 and PINK1-NFKB-4 showed limited ability in competing with NFkBwt-IR700 to bind with NFkB (Figure 3D, lane 10 and 14).

To further confirm the binding between PINK1-NFkB-1 in the human PINK1 promoter region and NFKB, we performed EMSA with IRDye-700 labelled PINK1-NFkB-1. The probe showed a shifted band (Figure 3E lane 2) after incubation with nuclear extracts from HEK293 cells overexpressing NFkB. The intensity of the shifted band was remarkably reduced when a 10 -fold excess of NFkB-Wtoligo was added to the incubation and abolished after the concentration of the competitor increased to 100-fold (Figure 3E, lane3-4). However, no competition was found when either 10-fold or 100-fold NFkB-Mu-oligo was added (Figure 3E, lane 5 and 6). A super-shift assay was further performed with the anti-p65 antibody. The supershifted band containing labelled PINK1-NFkB-1, NFkB, and anti-p65 antibody was observed at the top of the gel (Figure 3E, lane 7), and the band was competed down by NFkB-Wt-oligo (Figure 3E lane 8). Taken together, the EMSA results demonstrate that $\mathrm{NF} K \mathrm{~B}$ binds to the 
human PINK1 gene promoter and the -1493 to $-1474 \mathrm{bp}$ of PINK1 promoter region is the main binding site.

\section{NFKB increases the human PINK1 gene expression}

Next we examined the effects of NFKB on PINK1 gene expression by RT-PCR and Western blot analysis. The semi-quantitative RT-PCR was performed to test whether endogenous PINK1 mRNA level was affected by $N F \kappa B$. Using the human $\beta$-actin as an internal control, $N_{K} \mathrm{~B}$ overexpression significantly increased endogenous human PINK1 transcription by $59.51 \% \pm 15.13 \%(p<0.01)$ (Figure 4A). To examine whether NFKB modulates PINK1 expression at the protein level, endogenous PINK1 protein was detected by Western blot analysis. The full-length human PINK1 protein (FL-PINK1, $63 \mathrm{kDa}$ ) and its proteolytic products, $\Delta 1$-PINK1 $(55 \mathrm{kDa})$ and $\Delta 2$-PINK1 $(45 \mathrm{kDa})$, were detected and analyzed. The endogenous PINK1 expression increased by $114.53 \% \pm 38 \%(p<0.01)$ (Figure 4B) in the NFkB overexpressing HEK293 cells when compared with the vector control. Meanwhile, the same experiment was performed in SH-SY5Y cells and three PINK1 species were observed. Consistently, the endogenous PINK1 bands were also up-regulated in NFkB overexpressing SH-SY5Y cells by $45.26 \% \pm 19.58 \%$,
$41.10 \pm 6.70 \%$ and $48.54 \pm 4.50 \%(p<0.01)$, respectively (Figure 4C). Furthermore, when the NFkB activator LPS (Lipopolysaccharide) was used to treat SH-SY5Y cells for 16 hours, endogenous PINK1 protein levels were elevated compared to the untreated control by $26.77 \% \pm 2.33 \%$, $24.46 \pm 2.59 \%$ and $83.57 \pm 4.60 \%(p<0.001)$ (Figure $4 \mathrm{D})$, respectively.

Mitochondrial dysfunction plays a critical role in the development of PD and other neurodegenerative diseases such as Alzheimer's disease [26-29] and multiple sclerosis [30,31]. Mutations in several genes including UCHL1 [32], LRRK2 [33], PINK1 [12,34], PARKIN [35] are associated with PD pathogenesis. PINK1 is a mitochondrial targeted protein and has been extensively studied because of its crucial role in autosomal recessive familial Parkinson's disease [36] and protective role against mitochondrial dysfunction, oxidative stress and apoptosis $[37,38]$. However, the regulation of PINK1 gene expression has not been well addressed yet [39]. For the first time, we studied the transcriptional regulation of the human PINK1 gene in the present study.

To investigate the transcription and translation of the human PINK1 gene, we first mapped the adenine $91 \mathrm{bp}$ upstream of the first ATG in exon 1 as the transcription start site. Next we cloned the $1.8 \mathrm{~kb}$ fragment of 5' UTR


Figure 4 NFKB upregulates human PINK1 gene expression. (A) NFKB increases the endogenous human PINK1 mRNA level. HEK293 cells were transfected with either the NFKB expression vector or empty vector PMTF. RT-PCRs were performed using either primers specific to the human PINK1 coding sequence or the human $\beta$-actin coding sequence. (B) NFKB increases the endogenous human PINK1 protein levels. HEK293 cells transfected with NFKB were harvested $48 \mathrm{~h}$ after transfection for protein detection. Cell lysates were run on 10\% glycine gel and images were collected by Licor. A significant increase of endogenous PINK1 was observed. (C) The endogenous human PINK1 protein level was dramatically increased by NFKB in SH-SY5Y cells. SH-SY5Y cells were transfected with NFKB p65 plasmids or the control vector pMTF and then harvested for protein detections. The human PINK1 protein and its proteolysis products $\triangle 1$-PINK1 (55kD) and $\triangle 2$-PINK1 (45kD) were all increased. $\beta$-actin acted as the internal control. (D) LPS treatment facilitated PINK1 expression in SH-SY5Y cells. Cells were harvested after being treated for $16 \mathrm{~h}$ and then subjected to Western blot. The human $\beta$-actin level was served as a control. Quantification was performed by Image J software. Values indicate means \pm SEM. $n=3,{ }^{*} p<0.01$ by Student's $t$-test. 
of the human PINK1 gene into a promoterless vector upstream of a reporter luciferase gene. The luciferase assay showed that this $1.8 \mathrm{~kb}$ fragment has strong promoter activity, indicating that the $1.8 \mathrm{~kb}$ fragment functions as the human PINK1 gene promoter. Subsequently, by deletion analysis we found that the $104 \mathrm{bp}$ fragment from -78 to 26 serves as the minimal promoter region. The human PINK1 gene promoter does not have high GC content $(<50 \%)$ like the human BACE1 and TMP21 promoter $[40,41]$. Computer-based analysis revealed that the human PINK1 promoter contains several putative transcriptional factor binding elements such as MZF1, AP1F, CREB, NFKB and EGRF. Due to the importance of NFKB in PD, the potential regulation of PINK1 by NFKB was examined. There are four putative $\mathrm{NF}_{K} \mathrm{~B}$ binding sites in the human PINK1 gene promoter and our results indicate that two of them physically bind with $\mathrm{NF}$ B $\mathrm{p} 65$ with one strong binding site at the distal end. The effect of NFkB p65 on human PINK1 gene promoter was further confirmed by luciferase assay, where the overexpression of NFkB p65 elevated PINK1 promoter activity dramatically. Furthermore, we examined the effects of overexpression of NFKB p65 on PINK1 expression at the transcription and translation levels. Indeed, NFkB p65 increased the human PINK1 expression significantly at both the mRNA and protein levels. Due to the low expression level of the human PINK1 gene, few commercially available antibodies could detect the endogenous PINK1 clearly. We therefore cloned the human PINK1 plasmid, encoding a full-length PINK1 protein $(\sim 63 \mathrm{kDa})$ that is proteolytically processed into at least two shorter forms $(\sim 55 \mathrm{kDa}$ and $\sim 45 \mathrm{kDa})$, into a human dopaminergic neuroblastoma cell line (SHSY5Y) $[6,7,42]$. In this cell line, we were able to detect three PINK1-related bands. Only the full-length PINK1 could be detected in the HEK293 cell line. This might be due to different expression levels and mitochondrial function between the different cell lines.

In this study, we clearly showed that $N_{K} B$ significant upregulates PINK1 expression in both human and rodent cell lines, indicating that same regulation pathway exists in both human and rodents. It provides the strong evidence to support the use of rodent models to study the regulation of PINK1 expression in vivo, promoting the investigation of PD pathogenesis and drug development. Moreover, as SH-SY5Y cells are human dopaminergic cells and the dopaminergic neurons are mainly affected in PD patients, the results from SH-SY5Y cells can better represent the possible PINK1 regulation pathway in the brain of PD patients.

$\mathrm{N}$-terminal proteolysis of FL-PINK1 produces two fragments, $\Delta 1$-PINK1 and $\Delta 2$-PINK1. After processing the proteolysis productions translocate to cytosol and undergo proteasome degradation $[6,7,42]$. Although the functional role of PINK1 in mitochondria or in Parkinson's disease has not been well defined, PINK1 has been shown to confer a protective effect through preventing mitochondrial dysfunction, anti-apoptosis and promoting cell survival [43]. It has also been reported that FL-PINK1 accumulates in the brains of patients with sporadic PD or other synucleinopathies, and interestingly, the cleavage products of PINK1 are also increased in PD brains [44-46]. This accumulation of $\Delta$-PINK1 might due to the upregulation of PINK1 expression in response to PD-related stress [44]. Given that truncated PINK1 can confer protection, increased cleavage of PINK1 may play a role in protecting neurons during PD pathogenesis. Our result showed that all of the three forms of PINK1 (FL-PINK1, $\Delta 1$-PINK1 and $\triangle 2$-PINK1) were elevated by overexpression or activation of $\mathrm{NF}_{K} \mathrm{~B}$ signaling. Thus, the increase of PINK1 expression by NFKB activation could be neuroprotective. On the other hand, previous studies have suggested that the elevation of $\mathrm{NFKB}$ in dopaminergic neurons of PD patients is a reflection of the apoptotic and inflammatory state of the diseased brain [16]. These studies suggest that the activation of $\mathrm{NF}_{\mathrm{K} B}$ could be a double-edged sword in PD pathogenesis and the role of $\mathrm{NF}_{\kappa} \mathrm{B}$ dysregulation in $\mathrm{PD}$ warrants further study.

\section{Conclusion}

Our results demonstrate that PINK1 expression is tightly regulated at its transcription level and $\mathrm{NFKB}_{\mathrm{K}}$ signaling is a positive regulator for PINK1 gene expression.

\section{Methods}

\section{Primers and plasmid construction}

To amplify the 5'-untranslated region of the PINK1 gene from DNA extracted from the human neuroblastoma cells SH-SY5Y, a forward primer (5'-cgcgagctcgttgcccag gctggtcttg) corresponding to $-1799 \mathrm{bp}$ of the transcriptional start site on exon 1 and a reverse primer (5'-ccc aagcttcacaacaaacttggggcggtgcc) corresponding to $+26 \mathrm{bp}$ of the transcriptional start site were used. The PINK1 DNA fragment was cloned into a pGL3-basic vector upstream of a luciferase reporter gene (Promega) to construct pPINK1-A. Primers were designed to include restriction enzyme sites so that the PCR-amplified fragments can be inserted into the multiple cloning sites of the pGL3-basic vector. A series of deletion mutations (pPINK1-B, pPINK1-C, pPINK1-D, pPINK1-E, pPINK1-F, pPINK1-G, pPINK1-H and pPINK1-I) of PINK1 promoter were constructed utilizing primers listed as following: 26 F-cccaagcttcacaacaaacttggggcggtgcc, and 1799R- cccaa gcttgttgccaggctggtcttg; -795 F -ggcatggggatccaccatcttg, and -339 F-ccgctcgaggacctcgaatgctgccc; -103 F-ccgctcg agaaagtcactgctagaggc, and -50R-cccaagcttcgactcggcgcgt ggggg; -78 F-ccgctcgagccagcatagcgccccac, and $-47 \mathrm{~F}$ 
-ccgctcgaggaactgccgcgggggccg, and -19 F-ccgctcgagcca gcgcctgcgcctgcg, respectively.

\section{Switching mechanism at 5'end of RNA transcription (SMART) RACE cDNA amplification}

Total RNA was extracted from SH-SY5Y cells using TRI reagent (Sigma) following the manufacturer's protocol. SMART-RACE was performed using the SMARTer ${ }^{\text {TM }}$ RACE cDNA Amplification Kit (Clontech) following the user's manual. Simply, the first stand cDNA was synthesized from total RNA extracted from SH-SYHY cells with oligo $(\mathrm{dT})$ primer in the presence of SMARTer IIA oligonucleotide (5'-aagcagtggtatcaacgcagagtacxxxxx (X is undisclosed base in the proprietary SMARTer oligo sequence). The SMARTer IIA oligonucleotide is able to anneal to the 5 -end of the first stand CDNA and serve as template to extend the 5 '-end cDNA tail. A PINK1 reverse primer (5'-ccgaagcttgcctgcaagcgtctcgtgt) was specifically designed to recognize the +431 to +450 bp of human PINK1 gene downstream of the translation start site (ATG). The PCR products containing PINK1promoter region were amplified using SMARTer IIA oligonucleotide and PINK1 reverse primer and the first stand cDNA as template. The resulting PCR products were cloned into pcDNA4/myc-His A vector for sequencing and the first nucleotide linking with the adapter sequence was identified as the transcription start site of the human PINK1 gene.

\section{Cell culture, transfection, and luciferase assay}

Human embryonic kidney HEK293, mouse neuroblastoma N2A and human neuroblastoma SH-SY5Y cell lines were cultured in Dulbecco's modified Eagle's medium (DMEM) containing 10\% fetal bovine serum (FBS), $1 \mathrm{mM}$ sodium pyruvate, $2 \mathrm{mM}$ L-glutamine, 50 units $/ \mathrm{ml}$ penicillin G sodium, and $50 \mu \mathrm{g} / \mathrm{ml}$ streptomycin sulfate (Invitrogen). All cells were maintained in a humidified $37^{\circ} \mathrm{C}$ incubator containing $5 \% \mathrm{CO}_{2} .500 \mathrm{ng}$ plasmid DNA per well of 24-well-plate for luciferase assay or $2 \mu \mathrm{g}$ plasmid DNA per 35-mm-diameter plate for RNA extraction and Western blot analysis were used for cell transfection, respectively. For luciferase assay, pCMV-Rluc (Promega, USA) was co-transfected as a control to normalize the transfection efficiency. The cells were transfected using PEI reagent (Cat ${ }^{\#}$. 23966, Polysciences Inc.) or Lipofectamine $^{-{ }^{\mathrm{Im}}} 2000$ reagent (Invitrogen). Cells were harvested $24 \mathrm{hrs}$ after transfection and lysed with $100 \mu \mathrm{l}$ $1 \times$ passive lysis buffer (Promega) per well. Firefly luciferase activities and Renilla luciferase activities of the same sample were sequentially assayed on a luminometer (Turner Designs Model 20/20) following the protocol of the Dual-luciferase Reporter Assay System (Promega). The firefly luciferase activity was normalized to the Renilla luciferase activity and the resulted value reflected the promoter activity.

\section{Electrophoretic mobility shift assay (EMSA)}

HEK293 cells were transiently transfected with NFkB p65 and nuclear extracts were collected. Cells were rinsed and harvested with $1 \times$ phosphate-buffered saline. After centrifugation, cell pellets were resuspended with $5 \times$ volume of buffer A [10 mM HEPES pH 7.9, $10 \mathrm{mM} \mathrm{KCl}$, $0.1 \mathrm{mM}$ EDTA, $0.1 \mathrm{mM}$ EGTA, $1 \mathrm{mM}$ dithiothreitol (DTT), $0.5 \mathrm{mM}$ phenylmethylsulfonyl fluoride (PMSF)]. Cells were pipetted up and down gently and maintained on ice for 15 minutes. The cell suspension was transferred to a Kontes all glass Dounce tissue grinder and ruptured by 10 strokes. $10 \%$ NP40 was added into the cell suspension for a final concentration of $0.5 \%$. The samples were placed on ice for 15 minutes and stroked 5 more times. Crude nuclei were collected by centrifugation at $2000 \mathrm{~g}$ for 10 minutes and washed three times with buffer A containing $0.5 \%$ NP40 and resuspended in buffer $\mathrm{C}[20 \mathrm{mM}$ HEPES pH 7.9, 0.4 mM NaCl, 1 mM EDTA, 1 mM EGTA, $1 \mathrm{mM}$ dithiothreitol (DTT), $1 \mathrm{mM}$ phenylmethylsulfonyl fluoride (PMSF), 10\% Glycerol] at $4^{\circ} \mathrm{C}$ for $15 \mathrm{~min}$. The samples were centrifuged at $12000 \mathrm{~g}$ for $4 \mathrm{~min}$ at $4^{\circ} \mathrm{C}$, and the supernatant containing nuclear proteins was collected. EMSA was performed as previously described [40]. Four pairs of oligonucleotides containing the putative $\mathrm{NF}_{k} \mathrm{~B}$ binding site on human PINK1 promoter region were synthesized for detecting the binding ability of $N F \kappa B$ to PINK1 promoter. The sequences of the oligos were listed as following: NFKB-54-35, forward, agtcggggaactgccgcggg and reverse, cccgcggcagttcccgact; NFKB-111-92, forward, gcaaagggaaagtcactgct and reverse, agcagtgactttcctttgc; NFkB-814-794, forward, ggcatggggatccaccatctt and reverse aagatggtggatccccatgcc; NFkB-1493-1474, forward, caaatg ggaaattcatctat and reverse atagatgaatttcccatttg. The probes were labeled with IRDye-700 (IDT). Prior to incubation with nuclear extract, oligonucleotide probes were heated at $98^{\circ} \mathrm{C}$ for 5 minutes and annealed at $65^{\circ} \mathrm{C}$ for 10 minutes. $0.5 \mathrm{pmol}$ of annealed probes were incubated with $2 \mu \mathrm{l}$ of nuclear extract for 20 minutes at $22^{\circ} \mathrm{C}$ and the reaction mixtures were separated on a $4 \%$ Tris-glycine-EDTA gel in darkness. The mobility of probes on the gel was visualized using the LI-COR Odyssey (LI-COR Biosciences). For the competition assay, wild-type NFKB consensus oligonucleotides (forward: agttgaggggactttccaggc, and reverse: gcctgggaaagtccctcaact) and mutant NFKB consensus oligonucleotides (forward: agttgaggccactttccaggc, and reverse: gcctgggaaagtggcctcaact) were used as positive and negative controls, respectively. For the super shift assay, the nuclear extract was incubated in $10 \times$ EMSA binding buffer (100 mM Tris, $500 \mathrm{mM} \mathrm{KCl,} 10 \mathrm{mM}$ dithiothreitol, $\mathrm{pH}$ 7.5) with monoclonal anti-NFkBp65 antibody (Sigma) for 10 minutes prior to the probe adding. 


\section{Semi-quantitative RT-PCR}

HEK293 cells and SH-SY5Y cells transfected with NFkB p65 were harvested 48 hours after the transfection and total RNA was extracted using TRI reagent (Sigma). Reverse transcription was sequentially performed using ThermoScript ${ }^{\text {tw }}$ RT-PCR system kit following the manufacturer's protocol (Invitrogen). The PINK1 gene specific primer PINK1 776 F-taccagtgcaccaggagaag and PINK1 984R- gcttgggacctctcttggat were used to amplify a $208 \mathrm{bp}$ fragment of human PINK1 gene. The $\beta$-actin gene was also amplified using the forward primer (hActin662fBamHI: cgaggatccggacttcgagcaagagatgg) and reverse primer (hActin-1124rXbaI: cagtctagagaagcatttgcggtggacg), which produced a $500 \mathrm{bp}$ fragment. The PCR products were analyzed on $2 \%$ agarose gels.

\section{Western blot analysis}

Western blot was performed as described previously $[47,48]$. NFkB p65 plasmid DNAs $(2 \mu \mathrm{g} / 35 \mathrm{~mm}$ dish) were transfected into HEK293 cells and SH-SY5Y cells. 48 hours after transfection, cells were harvested by RIPA-DOC buffer (1\% Triton X-100, 0.1\% sodium dodecylsulphate, $1 \%$ sodium deoxycholate, $0.15 \mathrm{mM} \mathrm{NaCl}$, $0.05 \mathrm{M}$ Tris-HCl pH 7.2, $0.5 \mathrm{mM}$ phenylmethylsulfonyl fluoride) containing protease inhibitors (Roche). Cell lysates were separated on $10 \%$ Tris-glycine gel and proteins were transferred onto PVDF membrane, followed by primary antibody incubation overnight at $4^{\circ} \mathrm{C}$. The primary antibodies were rabbit anti-PINK1 polyclonal antibody (1:500 dilution, Novus BC100-494), anti-p65 (1:1000 dilution, Cell Signaling), monoclonal anti-NFKB p65 antibody (1:1000 dilution, Sigma), and anti- $\beta$-actin antibody AC-15 (1:100, 000 dilution, Sigma). The protein bands were visualized using the LI-COR Odyssey (LI-COR Biosciences) after secondary antibody (goat anti-mouse, goat anti-rabbit, LI-COR Biosciences) labeling. The resulted protein bands were quantified by Image J software. The experiment was done in triplicate to minimize experimental errors.

\section{Competing interests}

The authors declare that they have no competing interests.

\section{Authors' contributions}

XD and WS conceived and designed the experiments; XD, JT, QX, YW performed the experiments; XD, YW, TL and WS analyzed the data; FC, WS contributed reagents/materials/analysis tools; XD, YW and WS wrote the paper. All authors read and approved the final manuscript.

\section{Acknowledgements}

We thank Si Zhang, Juelu Wang, Yi Yang and Zhe Wang for providing valuable comments and technique supports. This work was supported by Canadian Institutes of Health Research (CIHR) Operating Grant TAD-117948 (W.S), and National Natural Science Foundation of China (NSFC) Grant 81161120498 (T.L.). WS is the holder of the Canada Research Chair in Alzheimer's Disease (Tier 1). X.D. is supported by the Chinese Scholarship Council awards.
Received: 4 June 2014 Accepted: 29 July 2014

Published: 11 August 2014

\section{References}

1. Van Den Eeden SK, Tanner CM, Bernstein AL, Fross RD, Leimpeter A, Bloch DA, Nelson LM: Incidence of Parkinson's disease: variation by age, gender, and race/ethnicity. Am J Epidemiol 2003, 157(11):1015-1022.

2. Parkinson J: An essay on the shaking palsy. 1817. J Neuropsychiatry Clin Neurosci 2002, 14(2):223-236. discussion 222

3. Shulman JM, De Jager PL, Feany MB: Parkinson's disease: genetics and pathogenesis. Annu Rev Pathol 2011, 6:193-222.

4. Lewy F: Paralysis agitans. I. Pathologische anatomie. Handbuch der Neurologie 1912, 3:920-933.

5. Unoki M, Nakamura Y: Growth-suppressive effects of BPOZ and EGR2, two genes involved in the PTEN signaling pathway. Oncogene 2001, 20(33):4457-4465.

6. Lin W, Kang UJ: Characterization of PINK1 processing, stability, and subcellular localization. J Neurochem 2008, 106(1):464-474.

7. Takatori S, Ito G, Iwatsubo T: Cytoplasmic localization and proteasomal degradation of N-terminally cleaved form of PINK1. Neurosci Lett 2008, 430(1):13-17.

8. Greene AW, Grenier K, Aguileta MA, Muise S, Farazifard R, Haque ME, McBride HM, Park DS, Fon EA: Mitochondrial processing peptidase regulates PINK1 processing, import and Parkin recruitment. EMBO Rep 2012, 13(4):378-385.

9. Fedorowicz MA, de Vries-Schneider RL, Rub C, Becker D, Huang Y, Zhou C, Alessi Wolken DM, Voos W, Liu Y, Przedborski S: Cytosolic cleaved PINK1 represses Parkin translocation to mitochondria and mitophagy. EMBO Rep 2014, 15(1):86-93.

10. Xiong H, Wang D, Chen L, Choo YS, Ma H, Tang C, Xia K, Jiang W, Ronai Z, Zhuang $X$, Zhang Z: Parkin, PINK1, and DJ-1 form a ubiquitin E3 ligase complex promoting unfolded protein degradation. J Clin Invest 2009, 119(3):650-660.

11. Morais VA, Verstreken $P$, Roethig A, Smet J, Snellinx A, Vanbrabant M, Haddad D, Frezza C, Mandemakers W, Vogt-Weisenhorn D, Van Coster R, Wurst W, Scorrano L, De Strooper B: Parkinson's disease mutations in PINK1 result in decreased Complex I activity and deficient synaptic function. EMBO Mol Med 2009, 1(2):99-111.

12. Valente EM, Abou-Sleiman PM, Caputo V, Muqit MM, Harvey K, Gispert S, Ali Z, Del Turco D, Bentivoglio AR, Healy DG, Albanese A, Nussbaum R, Gonzalez-Maldonado R, Deller T, Salvi S, Cortelli P, Gilks WP, Latchman DS, Harvey RJ, Dallapiccola B, Auburger G, Wood NW: Hereditary early-onset Parkinson's disease caused by mutations in PINK1. Science 2004, 304(5674):1158-1160.

13. Valente EM, Bentivoglio AR, Dixon PH, Ferraris A, lalongo T, Frontali M, Albanese A, Wood NW: Localization of a novel locus for autosomal recessive early-onset parkinsonism, PARK6, on human chromosome 1p35-p36. Am J Hum Genet 2001, 68(4):895-900.

14. Shembade N, Harhaj NS, Liebl DJ, Harhaj EW: Essential role for TAX1BP1 in the termination of TNF-alpha-, IL-1- and LPS-mediated NF-kappaB and JNK signaling. EMBO J 2007, 26(17):3910-3922.

15. Liu S, Chen ZJ: Expanding role of ubiquitination in NF-kappaB signaling. Cell Res 2011, 21(1):6-21.

16. Mogi M, Kondo T, Mizuno Y, Nagatsu T: p53 protein, interferon-gamma, and NF-kappaB levels are elevated in the parkinsonian brain. Neurosci Lett 2007, 414(1):94-97.

17. Hunot $S$, Brugg B, Ricard D, Michel PP, Muriel MP, Ruberg M, Faucheux BA, Agid Y, Hirsch EC: Nuclear translocation of NF-kappaB is increased in dopaminergic neurons of patients with parkinson disease. Proc Natl Acad Sci U S A 1997, 94(14):7531-7536.

18. Cao JP, Wang HJ, Yu JK, Liu HM, Gao DS: The involvement of NF-kappaB p65/p52 in the effects of GDNF on DA neurons in early PD rats. Brain Res Bull 2008, 76(5):505-511.

19. Ly PT, Wu Y, Zou H, Wang R, Zhou W, Kinoshita A, Zhang M, Yang Y, Cai F, Woodgett J, Song W: Inhibition of GSK3beta-mediated BACE1 expression reduces Alzheimer-associated phenotypes. J Clin Invest 2013, 123(1):224-235.

20. Chen CH, Zhou W, Liu S, Deng Y, Cai F, Tone M, Tone Y, Tong Y, Song W: Increased NF-kappaB signalling up-regulates BACE1 expression and its therapeutic potential in Alzheimer's disease. Int I Neuropsychopharmacol 2012, 15(01):77-90. 
21. Zheng L, Liu H, Wang P, Song W, Sun X: Regulator of calcineurin 1 gene transcription is regulated by nuclear factor-kappaB. Curr Alzheimer Res 2014, 11(2):156-164.

22. Wang R, Zhang M, Zhou W, Ly PT, Cai F, Song W: NF-kappaB signaling inhibits ubiquitin carboxyl-terminal hydrolase L1 gene expression. J Neurochem 2011, 116(6):1160-1170.

23. Wang K, Liu S, Wang J, Wu Y, Cai F, Song W: Transcriptional regulation of human USP24 gene expression by NF-kappa B. J Neurochem 2014, 128(6):818-828.

24. Pranski E, Van Sanford CD, Dalal N, Orr AL, Karmali D, Cooper DS, Gearing M, Lah JJ, Levey Al, Betarbet R: NF-kappaB activity is inversely correlated to RNF11 expression in Parkinson's disease. Neurosci Lett 2013, 547:16-20.

25. Hellweg CE, Baumstark-Khan C, Horneck G: Generation of stably transfected Mammalian cell lines as fluorescent screening assay for NF-kappaB activation-dependent gene expression. J Biomol Screen 2003, 8(5):511-521.

26. Wu Z, Zhu Y, Cao X, Sun S, Zhao B: Mitochondrial toxic effects of abeta through mitofusins in the early pathogenesis of alzheimer's disease. Mol Neurobiol 2014. (Epub ahead of printing) PMID:24710686.

27. Hauptmann S, Scherping I, Drose S, Brandt U, Schulz KL, Jendrach M, Leuner K, Eckert A, Muller WE: Mitochondrial dysfunction: an early event in Alzheimer pathology accumulates with age in $A D$ transgenic mice. Neurobiol Aging 2009, 30(10):1574-1586.

28. Reddy PH: Amyloid beta, mitochondrial structural and functional dynamics in Alzheimer's disease. Exp Neurol 2009, 218(2):286-292.

29. Aliev G, Palacios HH, Walrafen B, Lipsitt AE, Obrenovich ME, Morales L: Brain mitochondria as a primary target in the development of treatment strategies for Alzheimer disease. Int J Biochem Cell Biol 2009, 41(10):1989-2004.

30. Bonora M, De Marchi E, Patergnani S, Suski JM, Celsi F, Bononi A, Giorgi C, Marchi S, Rimessi A, Duszynski J, Pozzan T, Wieckowski MR, Pinton P: Tumor necrosis factor-alpha impairs oligodendroglial differentiation through a mitochondria-dependent process. Cell Death Differ 2014, 21:1198-1208.

31. Ghafourifar P, Mousavizadeh K, Parihar MS, Nazarewicz RR, Parihar A, Zenebe WJ: Mitochondria in multiple sclerosis. Front Biosci 2008, 13:3116-3126.

32. Leroy E, Boyer R, Auburger G, Leube B, Ulm G, Mezey E, Harta G, Brownstein MJ, Jonnalagada S, Chernova T, Dehejia A, Lavedan C, Gasser T, Steinbach PJ, Wilkinson KD, Polymeropoulos MH: The ubiquitin pathway in Parkinson's disease. Nature 1998, 395(6701):451-452.

33. Paisan-Ruiz $C$, Jain $S$, Evans EW, Gilks WP, Simon J, van der Brug $M$, Lopez de Munain A, Aparicio S, Gil AM, Khan N, Johnson J, Martinez JR, Nicholl D, Carrera IM, Pena AS, de Silva R, Lees A, Marti-Masso JF, Perez-Tur J, Wood NW, Singleton AB: Cloning of the gene containing mutations that cause PARK8-linked Parkinson's disease. Neuron 2004, 44(4):595-600.

34. Valente EM, Salvi S, lalongo T, Marongiu R, Elia AE, Caputo V, Romito L, Albanese A, Dallapiccola B, Bentivoglio AR: PINK1 mutations are associated with sporadic early-onset parkinsonism. Ann Neurol 2004, 56(3):336-341.

35. Kitada T, Asakawa S, Hattori N, Matsumine H, Yamamura Y, Minoshima S, Yokochi M, Mizuno Y, Shimizu N: Mutations in the parkin gene cause autosomal recessive juvenile parkinsonism. Nature 1998, 392(6676):605-608.

36. Criscuolo C, Volpe G, De Rosa A, Varrone A, Marongiu R, Mancini P, Salvatore E, Dallapiccola B, Filla A, Valente EM Criscuolo C, Volpe G, De Rosa A, Varrone A, Marongiu R, Mancin P, Salvatore E, Dallapiccola B, Filla A, Valente EM, De Michele G: PINK1 homozygous W437X mutation in a patient with apparent dominant transmission of parkinsonism. Movement disorders: official journal of the Movement Disorder Society 2006, 21(8):1265-1267.

37. Gautier CA, Kitada T, Shen J: Loss of PINK1 causes mitochondrial functional defects and increased sensitivity to oxidative stress. Proc Nat/ Acad Sci U S A 2008, 105(32):11364-11369.

38. Wang X, Winter D, Ashrafi G, Schlehe J, Wong YL, Selkoe D, Rice S, Steen J, LaVoie MJ, Schwarz TL: PINK1 and Parkin target Miro for phosphorylation and degradation to arrest mitochondrial motility. Cell 2011, 147(4):893-906.

39. Tufi R, Gandhi S, de Castro IP, Lehmann S, Angelova PR, Dinsdale D, Deas E, Plun-Favreau H, Nicotera P, Abramov AY Willis AE, Mallucci GR, Loh SH, Martins LM: Enhancing nucleotide metabolism protects against mitochondrial dysfunction and neurodegeneration in a PINK1 model of Parkinson's disease. Nat Cell Biol 2014, 16(2):157-166.

40. Christensen MA, Zhou W, Qing H, Lehman A, Philipsen S, Song W: Transcriptional regulation of BACE1, the beta-amyloid precursor protein beta-secretase, by Sp1. Mol Cell Biol 2004, 24(2):865-874.
41. Liu S, Zhang S, Bromley-Brits K, Cai F, Zhou W, Xia K, Mittelholtz J, Song W: Transcriptional regulation of TMP21 by NFAT. Mol Neurodegeneration 2011, 6:21.

42. Deas E, Plun-Favreau H, Gandhi S, Desmond H, Kjaer S, Loh SH, Renton AE, Harvey RJ, Whitworth AJ, Martins LM, Abramov AY, Wood NW: PINK1 cleavage at position $\mathrm{A} 103$ by the mitochondrial protease PARL. Hum Mol Genet 2011, 20(5):867-879.

43. Sanchez-Mora RM, Arboleda H, Arboleda G: PINK1 overexpression protects against C2-ceramide-induced CAD cell death through the PI3K/AKT pathway. J Mol Neurosci 2012, 47(3):582-594.

44. Muqit MM, Abou-Sleiman PM, Saurin AT, Harvey K, Gandhi S, Deas E, Eaton S, Payne Smith MD, Venner K, Matilla A, Healy DG, Gilks WP, Lees AJ, Holton J, Revesz T, Parker PJ, Harvey RJ, Wood NW, Latchman DS: Altered cleavage and localization of PINK1 to aggresomes in the presence of proteasomal stress. J Neurochem 2006, 98(1):156-169

45. Gandhi S, Muqit MM, Stanyer L, Healy DG, Abou-Sleiman PM, Hargreaves I, Heales S, Ganguly M, Parsons L, Lees AJ, Latchman DS, Holton JL, Wood NW, Revesz T: PINK1 protein in normal human brain and Parkinson's disease. Brain 2006, 129(Pt 7):1720-1731.

46. Murakami T, Moriwaki Y, Kawarabayashi T, Nagai M, Ohta Y, Deguchi K, Kurata T, Morimoto N, Takehisa Y, Matsubara E, Ikeda M, Harigaya Y, Shoji M, Takahashi R, Abe K: PINK1, a gene product of PARK6, accumulates in alpha-synucleinopathy brains. J Neurol Neurosurg Psychiatry 2007, 78(6):653-654

47. Qing H, Zhou W, Christensen MA, Sun X, Tong Y, Song W: Degradation of BACE by the ubiquitin-proteasome pathway. Faseb J 2004, 18(13):1571-1573.

48. Zhang Z, Nadeau P, Song W, Donoviel D, Yuan M, Bernstein A, Yankner BA: Presenilins are required for gamma-secretase cleavage of beta-APP and transmembrane cleavage of Notch-1. Nat Cell Biol 2000, 2(7):463-465.

doi:10.1186/s13041-014-0057-y

Cite this article as: Duan et al:: Upregulation of human PINK1 gene expression by NFKB signalling. Molecular Brain 2014 7:57.

\section{Submit your next manuscript to BioMed Central and take full advantage of:}

- Convenient online submission

- Thorough peer review

- No space constraints or color figure charges

- Immediate publication on acceptance

- Inclusion in PubMed, CAS, Scopus and Google Scholar

- Research which is freely available for redistribution

Submit your manuscript at www.biomedcentral.com/submit
C) Biomed Central 